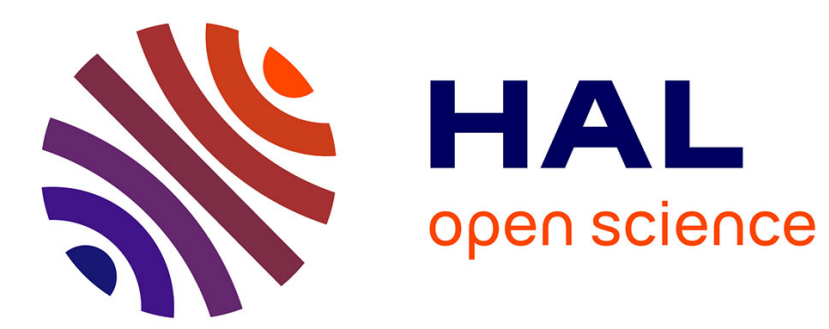

\title{
L'humanitaire militaire et la crise malienne (2012-2015). Vers une théorie de la crise complexe
}

Fabienne Le Houerou

\section{To cite this version:}

Fabienne Le Houerou. L'humanitaire militaire et la crise malienne (2012-2015). Vers une théorie de la crise complexe. Relations internationales, 2016, 165 (1), pp.97-116. 10.3917/ri.165.0097 . hal01374745

\section{HAL Id: hal-01374745 \\ https://hal.science/hal-01374745}

Submitted on 1 Oct 2016

HAL is a multi-disciplinary open access archive for the deposit and dissemination of scientific research documents, whether they are published or not. The documents may come from teaching and research institutions in France or abroad, or from public or private research centers.
L'archive ouverte pluridisciplinaire HAL, est destinée au dépôt et à la diffusion de documents scientifiques de niveau recherche, publiés ou non, émanant des établissements d'enseignement et de recherche français ou étrangers, des laboratoires publics ou privés. 


\title{
L'humanitaire militaire et la crise malienne (2012-2015). Vers une théorie de la crise complexe
}

\begin{abstract}
Cet article tente d'explorer le caractère de chronicité de la crise malienne (2012-2015) à la lumière du passé historique de ce pays. La gestion par la France de cette situation passe par une stratégie de communication moderne qui associe la dimension humanitaire à l' expérience militaire. En effet, il est question de présenter aux média le passage militaire comme un des éléments d'une ambition pacifique plus ambitieuse et plus «noble », telle celle de voler au secours d'un pays ami en difficulté. La crise malienne, à l'instar d'autres crises africaines, comme celle du Darfour, trouve ses racines dans l'histoire de la longue durée. Ce registre d'historicité et la récurrence d'affrontements " ethniques », sur des temps longs, sont des éléments caractéristiques des crises complexes et durables. L'auteur s'inspire des travaux d'Edgar Morin pour caractériser la question malienne comme le paradigme de la crise complexe et chronique. Il s'agit de flambée « endémique »- modèle paradoxal de convulsion permanente. La cessation de l'embrasement militaire et des hostilités armées ne correspond pas à une véritable extinction d'incendie ni à de véritables accords de paix mais à des solutions pacifiques précaires et fragiles susceptibles de s'enflammer à nouveau.
\end{abstract}

This paper is an attempt to explore the chronic dimension of the crisis in Mali (2012-2015) highlighting the past and the History of this country. The French way to handle the situation foster a modern communication strategy associating the humanitarian dimension to the military experience. The military adventure was presented to the media as a limited element of a wider and more noble and pacific ambition like assisting a friendly country in trouble. The war in Mali, like in others African crises, such as Darfur, has its roots causes in the long term history. This category of temporality added up with recurring ethnical confrontations, on long period of time, are typical of what we can consider a complex and durable crisis. The author is inspired by Edgar Morin theory to distinguish the crisis in Mali as a paradigm of a complex and chronic crisis. It is a question of endemic blaze (outburst)- a paradox model of permanent convulsion (spasm). The cessation of the military conflagration (blazing up) and the ending of armed hostilities do not meet a real extinction of the fire nor does it lead to real peace agreements but to fragile and uncertain pacific solutions that can inflame again and again.

Une crise est censée correspondre à un pic avec des seuils et former ontologiquement une courte parenthèse. Depuis 1959, l'Afrique nous force à constater que les états de crise ne sont pas des situations transitoires mais peuvent être endémiques et durables, traînant en longueur et en sourdine, hors champs des feux de l'actualité, oubliées par les univers médiatiques et donnant souvent l'illusion d'avoir trouvé leur solution à un public qui estime que le départ des armées et la fin du «moment militaire » consacrent la sortie de crise.

En réalité le départ des troupes coïncide souvent avec le chaos social et politique. De nombreux analystes estiment que le moment militaire ne fait qu'aggraver ou renforcer une crise car il ne donne que des réponses exogènes temporaires. Depuis les années 1990 et la 
politique de «Responsability to Protect $»^{1}$, les interventions militaires ont été présentées en Occident comme des réponses à des crises humanitaires, instaurant ainsi une confusion progressive entre militaire et humanitaire et inventant l'illusion d'un militaire humanitaire. La carte africaine dans le jeu des nations est éminemment sécuritaire. Elle se joue sur un échiquier où le terrorisme est depuis 2001 le problème majeur des chancelleries occidentales. Les crises internationales sont gérées par des institutions telles que la Communauté économique des États de l'Afrique de l'Ouest (Cédéao) ${ }^{2}$, l'ONU et l'Union africaine (UA) qui sont devenues des organes politiques. Contrer le jihadisme et le radicalisme islamiste est le pilier d'une diplomatie américaine qui oppose de façon binaire un "soft islam » (les frères Musulmans) à un «islam hard» (le jihadisme). Aussi le risque terroriste demeure-t-il le nœud gordien des politiques étrangères de puissances telles que les États-Unis ou la France, soucieuses de protéger des intérêts économiques et l'accès aux matières premières mais aussi de verrouiller l'accès à des territoires et de faire échec à ce qu'on pourrait appeler l'effet papillon du jihadisme, agent diffuseur des pollens du radicalisme sur la scène internationale. Il n'en demeure pas moins que c'est souvent sur des prétextes humanitaires que les États occidentaux interviennent en jouant au bon samaritain venu à la rescousse de populations en danger alimentaire et sécuritaire. La crise malienne n'a pas dérogé à ce modèle de travestissement du loup interventionniste en agneau.

Quelles sont les conséquences de ces glissements sémantiques et des bals masqués diplomatiques ? L'euphémisation de la violence est-elle réellement efficace dans la manière dont nos sociétés perçoivent les crises? Cet article entend analyser l'usage de l'humanitaire comme stratégie de communication. En effet, évoquer une intervention humanitaire pour désigner une attaque aérienne ou une opération militaire sur le sol d'un pays étranger fait partie d'un mode de communication occidental inspiré par la philosophie de la non-violence. Une guerre est d'autant mieux acceptée des opinions occidentales qu'elle est «vendue » comme une opération humanitaire. Ces précautions de langage sont-elles réellement efficaces? Les pays africains partenaires de la France sont-ils dupes ? Cet article tentera de répondre à ces questions en mettant en lumière le paradigme militaire-humanitaire de la France dans ses relations internationales à la lumière de la crise malienne. De quelle manière l'opération «Serval» a-t-elle été présentée par les médias français ? Quelles sont les déclencheurs immédiats et les causes profondes de la crise malienne? L'humanitaire n'est-il pas dans cette perspective une nouvelle carte dans le jeu de la Françafrique et ne pose-t-il pas comme élément de continuité ${ }^{3}$ ?

\footnotetext{
1. Le concept de « responsabilité de protéger » a été soutenu par 191 pays dans une résolution adoptée par le Sommet mondial des Nations Unies de 2005 (A/RES/60/1). Ce texte rappelle que chaque État est tenu de protéger sa population des génocides, crimes de guerre, épurations ethniques et crimes contre l'humanité. Lorsqu'un État n'assure pas la protection de sa population, abdiquant par conséquent sa responsabilité d'État souverain, et que les actions pacifiques échouent, la communauté internationale est tenue de prendre des mesures pour remédier à la situation, l'intervention militaire étant un dernier recours.

${ }^{2}$. Organisation intergouvernementale ouest-africaine créée le 28 mai 1975.
}

\footnotetext{
${ }^{3}$. Le terme «Françafrique », utilisé surtout pas les médias, est péjoratif car il suggère une forme de résurgence néocoloniale dans l'action de la France en Afrique. Il fait référence à une influence française plus au moins «occulte» qui se fonde sur un ensemble de relations complexes : réseaux d'influence, mécanismes politiques, économiques et militaires qui lient la France à ses anciennes colonies du continent ainsi qu'à quelques autres pays africains. Cette influence de l'ancienne puissance coloniale est parfois largement amplifiée par les organes de presse et les télévisions qui ont tendance à effectuer une lecture « complotiste» d'un ensemble diplomatique qui s'inscrit sur deux siècles et, qui, au-delà de ces caricatures, prend racine dans une histoire commune également tissée de solidarités et de dialogues.
} 
La présente analyse s'organise en deux parties. La première évoquera les motifs de l'intervention française et les stratégies imagétiques de communication en amont de la dite intervention, les raisons avancées par les acteurs étatiques et les ressorts économiques de la «diplomatie Areva» dans la région sahélo-saharienne. Dans un second temps, afin d'interroger la théorie qui prévaut dans les cercles géostratégiques relative à l'équivalence entre crise et carence de l'État postcolonial, seront rappelées les causes profondes de cette crise malienne de 2012-2015 au travers d'un bref retour sur les empires précoloniaux du Mali, États forts et structurés, et l'administration rationnelle qu'ils ont apportée aux territoires de la région. Ces rappels, présentés de façon volontairement succincte, sont, dans cette analyse, des repères essentiels. Nous conclurons sur les grands changements apportés par la période coloniale au Soudan français (actuel Mali) en termes de rationalité administrative et de rupture avec les traditions de contrôle des élites traditionnelles. Ces combinaisons entre lointain passé et histoire immédiate nous permettront finalement d'élaborer une théorie de la crise complexe en contrepoint des théoriciens des États faillis (Failed States) et d'envisager une mécanique d'emboîtement qui se met en place après la chute du mur de Berlin et la fin de la Guerre froide, et qui entretient un feu mal éteint, susceptible de reprendre continuellement. Une crise complexe se caractérise par une chronicité et une capacité à se modifier dans le temps. C'est le cas du Rwanda, du Congo, de l'Afghanistan, de la Somalie, du Tibet et, plus récemment, du Darfour. La crise malienne comporte elle aussi tous les ingrédients du paradigme de la crise complexe pluridimensionnelle telle que formulée par Edgar Morin ${ }^{4}$.

\section{LE DÉROULEMENT DE LA CRISE MALIENNE}

L'histoire immédiate nous rappelle que la crise fait irruption lorsque s'opère la jonction entre des groupes militaires touaregs et les combattants islamistes. Le Mouvement national de libération de l'Azawad (MNLA), Ansar El Din et les organisations islamistes issues d'AlQaïda au Maghreb islamique (Aqmi) s'emparent du Nord du Mali sur fond d'effondrement des élites maliennes et de l'État libyen ${ }^{5}$. La disparition de Kadhafi en 2011 provoque une lame de fond sur tous les pays voisins. La zone sahélo-saharienne avec le Tchad, la Mauritanie, le Niger fut dès lors le théâtre de recompositions militaires importantes impliquant divers groupes armés. Pôle d'attraction de la région, l'économie libyenne a aimanté de nombreux migrants maliens et nigériens. Dans les années 1970, les dirigeants touarègues ont bénéficié d'avantages pluriels en Libye, où ils forment un groupe de réfugiés politiques originaires du Mali et du Niger appelés Ishumar (chômeurs). À l'époque, Kadhafi s'employait à instrumentaliser la revendication politique identitaire touarègue. Les Ishumars, conscients de cette manipulation, n'ont jamais été les jouets naïfs du dictateur libyen et se sont éloignés de leur bienfaiteur dans les années 1990. Toutefois, la disparition définitive du créateur de la Jamahirya libyenne entraîna une redistribution des cartes aboutissant à la sécession du Nord du Mali. L'éclatement de l'État malien et sa disparition annoncée intervient au moment où les Touarègues s'allient aux islamistes dans une conquête territoriale qui culmine dans la proclamation de l'indépendance de l'Azawad (Mali du Nord) le 6 avril $2012^{6}$. Son territoire, en majeure partie désertique, englobe des zones saharienne et sahélienne, un espace historique dont les Touaregs, encore sous administration française, réclament l'indépendance depuis 1958 et le point de fixation de différentes rebellions touarègues depuis 1960. Une nouvelle insurrection est déclenchée en

\footnotetext{
4. Edgar Morin, « Pour une crisiologie », Communications, 1976, n² 25, pp. 149-163.

5. On peut traduire Anșār ad-Dīn par «défenseurs de la religion ». Aqmi : en arabe : القاعدة في بلاد المغرب

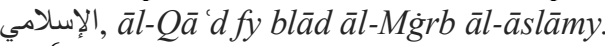

6. Azawad, ou Azaouad ou Azaouâd : ॰Ж⿻丷^^ en tifinagh, أزواد en arabe.
} 
janvier 2012 par le MNLA réclamant l'indépendance de l'intégralité des trois régions du nord du Mali, celles de Kidal, de Tombouctou et de Gao.

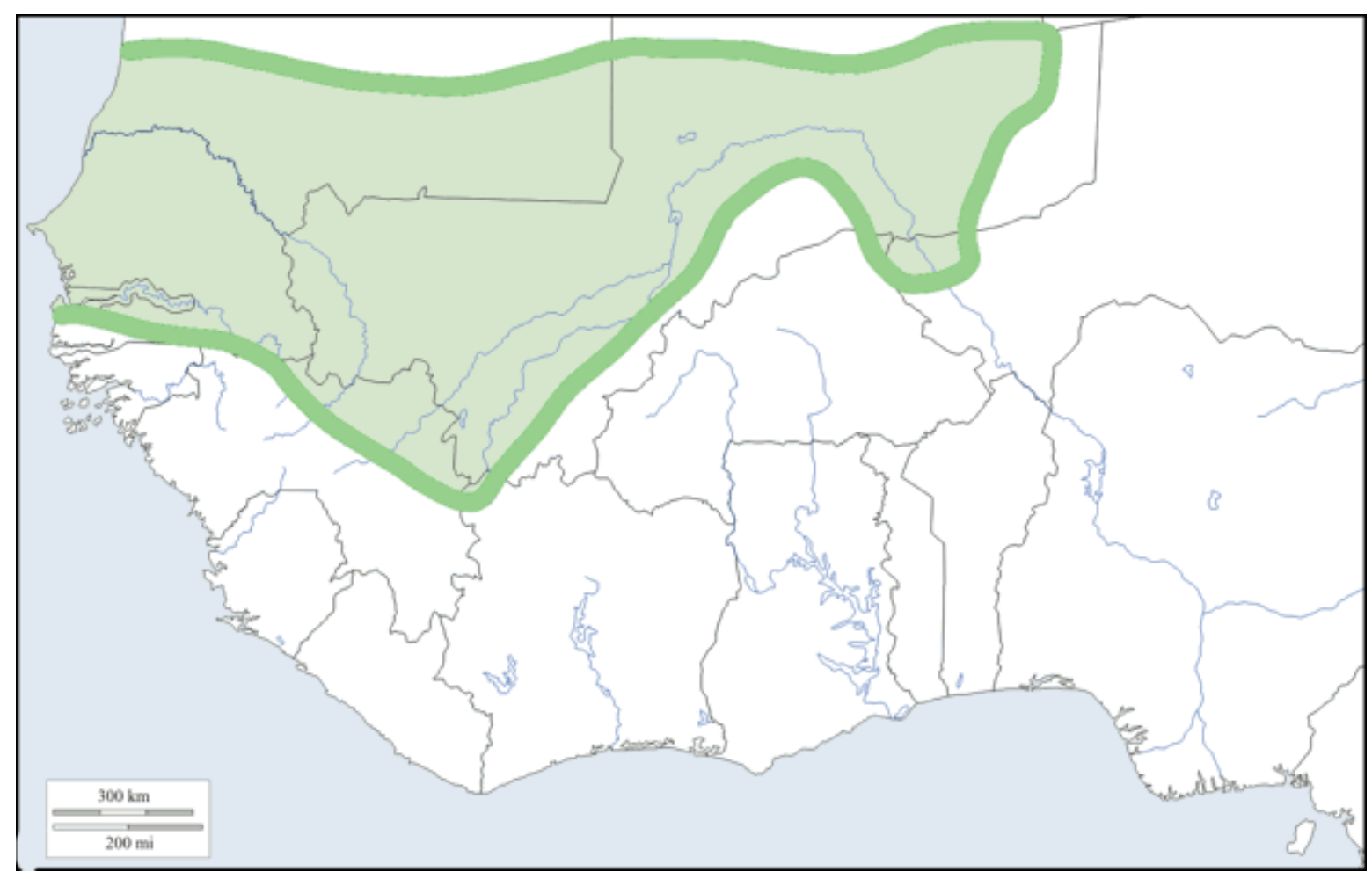

Fig.1 : L'empire du Mali au début du XIVe siècle. Crédit photographique Agathe Plauchut

Les événements de 2012 s'inscrivent donc dans une continuité historique et dans la logique d'une exclusion politique touarègue qui existe depuis la colonisation française du Soudan (Mali colonial). Aussi est-il pertinent de rappeler que les racines de la crise demeurent la colonisation, le tracé des frontières, la sous-administration et la marginalisation historique du Nord Mali, la relégation, la ségrégation et la paupérisation des minorités touarègues depuis la période coloniale. Ces soixante ans de déclin économique et social sur fond de revendication nationale inaudible ont façonné un imaginaire d'exil et des revendications territoriales et identitaires. À bien des égards, le «furoncle» touarègue et sa lente inflammation est à comprendre sur ce moyen. Cette période historique de 60 ans se présente comme une brève parenthèse.

Mais plusieurs acteurs agissent de façon emboîtée dans l'espace sahélien, faisant de la crise malienne une crise complexe où le long terme s'imbrique au court terme et où différents intérêts de groupes, fondamentalement opposés sur de nombreux points, conjuguent leurs efforts en vue d'un objectif commun : renverser le régime malien mais surtout casser l'édifice politique tout entier et rayer le Mali comme entité nationale. Cette conjonction de forces met en péril l'équilibre de cette région du monde et oblige les puissances occidentales à réagir pour des motifs sécuritaires et économiques. Areva ne demeure-t-elle pas la clef de voûte avouée - de l'action diplomatique et militaire française ? La France intervient militairement sur le terrain du 11 janvier 2013 au $1^{\text {er }}$ août 2014, sous mandat de l'ONU (résolution 2085), dans le cadre de l'opération «Serval». En février 2012, 4000 soldats français et 6000 soldats africains dont 2400 Tchadiens sont sur place. Les combattants de la partie adverse les forces conjuguées des mouvements Ansar Al Din, Aqmi, Mujao et Boko Haram avec une centaine de combattants - sont évalués à 3 000. Leur agenda déclaré est d'établir un État islamique fondé sur la Charia. Le bilan affiché des combats est de 10 morts français, 75 
victimes maliennes, 36 tchadiennes et 600 combattants salafistes tués ${ }^{7}$. Il s'agit donc d'un Blitzkrieg ("guerre éclair »), d'une offensive visant à emporter une victoire décisive par un engagement localisé et limité dans le temps, une opération coup de poing porteuse d'un immense déséquilibre militaire sur le terrain, qui est soutenue par un arsenal diplomatique faisant de la Cédéao, de l'ONU et de l'Union Africaine (UA) les piliers de leur stratégie. La crise répond également parfaitement au modèle du «Responsability to Protect» qui règle l'habillage juridique de l'intervention afin de lui donner une présentabilité et une respectabilité internationale. La presse a abondamment relayé les déclarations du président Hollande ainsi que celles de son gouvernement. Il était question de «voler au secours de nos amis maliens ». Le Nouvel Observateur justifiait l'intervention comme suit :

Elle [la France] l'a fait à la demande du président malien, Dioncounda Traore, qui cherche à repousser une offensive des rebelles du nord : ils avaient capturé la ville de Konna jeudi et menaçaient de prendre Mopti (100 000 habitants). Avec l'appui de la France, l'armée malienne a repris Konna ${ }^{8}$.

Cette justification, loin d'être fallacieuse ou erronée, était une réponse à une menace réelle visant le président malien, son gouvernement mais surtout la nation malienne qui, soumise au rythme des conquêtes territoriales réalisées par des groupes dits « rebelles », était susceptible d'éclatement.

Sauver l'État malien, tel était le leitmotiv des médias et des réseaux sociaux en 2012. Des images des exactions d'Aqmi, du Mouvement pour l'unicité et le jihad en Afrique de l'Ouest (Mujao) ${ }^{9}$, d'Ansar El Din et du MNLA ont largement circulé sur les télévisions du monde entier, mais c'est surtout sur internet (Twitter et Facebook) que des images, parfois truquées, faisaient étalage des atrocités perpétrées par les jihadistes au Nord Mali. La politique des images permet de travailler en amont la perception des publics occidentaux et de les préparer à une autojustification. Le message visuel insistait largement sur les sévices subis par les populations maliennes de Gao, Tombouctou et Kidal, des gros plans mettant l'accent sur la barbarie à l'œuvre des tenants d'un État islamique au Nord Mali. Les émotions suscitées par les images permettent de préparer l'opinion à accepter l'intervention militaire avant même qu'elle ne débute. Inonder l'espace médiatique de représentations des sévices corporels qui frappent l'imaginaire occidental permet, le moment venu, d'influencer les perceptions des publics afin qu'ils appréhendent la prise d'armes avec soulagement. De fait, sur les réseaux sociaux les commentaires des internautes ont de plus en plus largement exprimé une condamnation morale de la violence perpétrée par les forces indépendantistes du Nord Mali.

\section{L'insistance sur les mutilations}

Le conflit a débuté par un flot d'images des mutilations commises par le MNLA, Aqmi et le Mujao. Le principal flux de clichés montrait des pieds et de mains amputés. Une photo exposant quatre hommes qui tendaient leurs membres mutilés a eu un impact important. Postée sur les réseaux sociaux en 2012, elle a soulevé une vague d'émotion. Il s'agissait en réalité d'une photo prise au Soudan qui n'avait rien à voir avec le Mali, copiée sur un blog de propagande et réutilisée sur les réseaux sociaux lors de l'avancée des jihadistes au nord Mali

\footnotetext{
7. Le Point, 23 mars 2013, référence web : http://www.lepoint.fr/monde/mali-quelque-600-islamistes-tuesdepuis-janvier-27-03-2013-1646649_24.php. Page consultée le 12 septembre 2015.

8. http://rue89.nouvelobs.com/2013/01/12/pourquoi-la-france-entre-en-guerre-au-mali-238534. Page consultée le 12 octobre 2015.

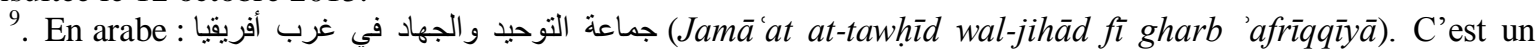
groupe armé djihadiste salafiste.
} 
afin de dénoncer leurs exactions ${ }^{10}$. Ces manipulations sont très fréquentes sur internet, en particulier sur les réseaux sociaux. Des montages photographiques accolent des clichés de sources et de périodes différentes. Une photo prise en 1999 au Soudan sera présentée comme un cliché du Mali en 2012. Ces collages, diffusés sur les réseaux sociaux, bouleversent la circulation et la hiérarchisation de l'information traditionnelle. L'absence de toute réglementation, de recoupement et de référencement permet de nombreuses falsifications.
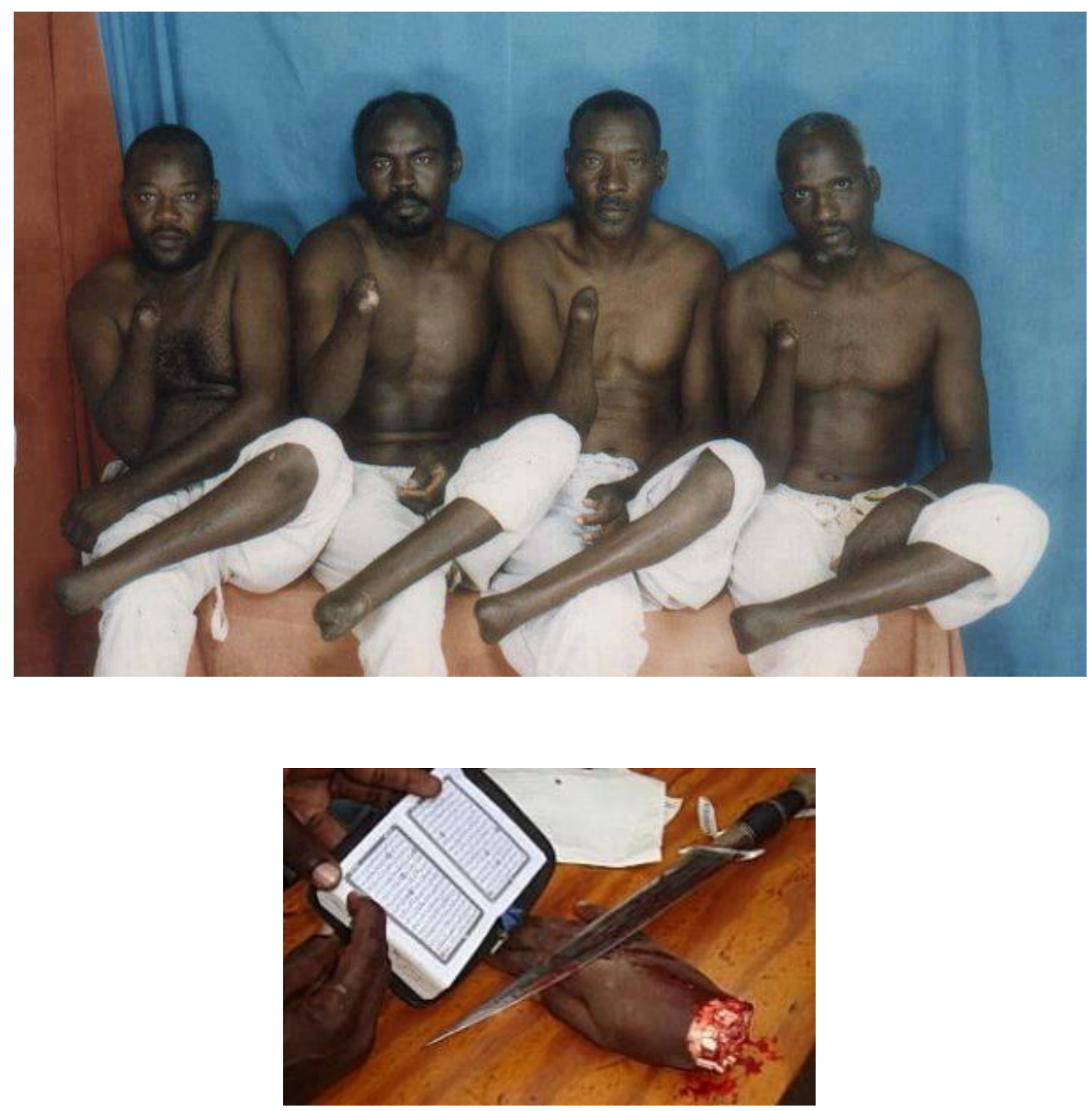

Fig. 2 : Photographies censées prises au Mali et publiées sur le blog : http://info.charia.overblog.com/ la-charia-c-est-aussi-l-amputation-des-mains-et-des-pieds, consulté le 18 janvier 2016.

10. Voir le site http://info.charia.overblog.com/page/2, qui met en ligne des images manipulées et des indications erronées sur leur provenance et leur datation. Page consultée le 12 octobre 2015. 
La propagande sur Facebook utilise ces contrefaçons pour susciter des réactions émotionnelles. Des groupes d'activistes de provenances très diverses cherchent à peser sur l'opinion en publiant des images choquantes, qui provoquent des rejets ou des sentiments exacerbés, voire des réactions d'horreur. Les groupes se réclamant de plateformes hostiles à l'islam se sont spécialisés, par exemple, dans des montages photos insistant sur les châtiments corporels particulièrement cruels dictés par la charia en pays musulmans. La chaîne de télévision France 3 a diffusé un document sur les amputations pratiquées par le Mujao à Gao, le journaliste se targuant de la «rareté » de ce genre d'images. Son reportage de deux minutes trente parvient à introduire une intensité narrative dans l'évocation de la violence et de la sévérité des châtiments en commençant durant la première minute par les coups de fouets administrés par les combattants du Mujao, pour terminer, au bout d'une minute et quarantesept secondes de reportage, sur des témoignages de voleurs amputés, point culminant du document. Ces derniers, interviewés à l'hôpital de Gao, dénoncent «cette charia-là » et expliquent comment ils ont été ligotés puis leurs membres tranchés, cela à huit secondes de la fin du reportage ${ }^{11}$. En deux minutes et demie, tout est dit de la barbarie des combattants. Des témoignages de réfugiés sur les violences sexuelles perpétrées par le MNLA ont été également diffusés, dont des témoignages de femmes de Gao et de Kidal sur Youtube ${ }^{12}$. L'analyse imagétique traduit une dynamique narrative qui met en place un dispositif d'abominations venant légitimer la prise d'arme, et les secours apportés aux populations torturées par les jihadistes.

La mise en scène de la famine (au Biafra, en Éthiopie, en Somalie) est devenue, depuis les années 1970, une construction de propagande très classique. L'affichage des souffrances des populations autochtones présente l'intervention comme une libération des populations. Cette technique médiatique nous vient des premiers journaux télévisés de l'époque coloniale, notamment ceux de L'Istituto Luce pendant la guerre d'Éthiopie, en 1936 où l'agresseur italien est toujours présenté en libérateur. Aussi le dispositif filmé de la presse française renvoie-t-il globalement aux systèmes d'information en vigueur en Occident depuis les débuts du cinéma et des actualités télévisées et reproduit-il un schéma bien rodé de communication audiovisuelle. Il convient de souligner cette singulière continuité de 1930 à nos jours, malgré les formidables progrès technologiques en matière d'enregistrement numérique des images. La méthode consiste à exposer les données de façon binaire et manichéenne, comme en témoigne la carte diffusée sur un grand média français qui sépare le Mali en deux grandes entités de population, essentialisant et caricaturant ainsi les rivalités ethniques, et invoquant un racisme des «Arabes et [des] Blancs», soit les Arabo-Touarègues, contre des Subsahariens noirs. Ce schéma médiatique est très semblable à celui déployé pendant la crise du Darfour (2003-2009), qui opposait «d'innocents » Noirs à de «cruels » janjawids, des Arabes racistes. Ces approches simplistes ne s'inspirent cependant pas d'un didactisme tentant d'expliquer à un public étranger des réalités sociales complexes dans des territoires inconnus. L'absence de nuances permet en réalité de justifier les politiques conduites par les chancelleries étrangères.

Le gouvernement français justifia l'intervention ainsi : il fallait «voler au secours » de nos amis malmenés, voire martyrisés, par la collusion islamo-touarègue. Le but de l'intervention militaire était présenté, d'une part, comme éminemment charitable envers le

11. Vidéo particulièrement intéressante car elle réunit le message essentiel divulgué tant pas la presse française que les réseaux sociaux sur la barbarie jihadiste : https://www.youtube.com/watch?v=fUQcGFteu5M. Page consultée le 12 septembre 2015.

${ }^{12}$. http://maliactu.net/exactions-du-mnla-au-nord-mali-temoignages-de-refugies-en-mauritanie/. Voir les nombreuses vidéos postées sur Youtube : https://www.youtube.com/watch?v=CxqiGdRLqrs ; https://www.youtube.com/watch?v=CxqiGdRLqrs ; https://www.youtube.com/watch?v=yJYiVcL_NWs. Pages consultées le 12 septembre 2015. 
Mali, pays ami, et comme une urgence pour sécuriser et protéger les ressortissants français, d'autre part. Ce renversement de message (proposer le militaire en sauveur et en bienfaiteur) est un élément remarquable de continuité depuis les premières actualités télévisées des années 1930 en Europe.

\section{Les objectifs officiels de la France}

Le rapport publié en mars 2013 par le Groupe de recherche et d'information pour la paix et la sécurité (GRIP) rapporte les propos que Jean-Marc Ayrault avait tenus devant les députés de l'Assemblée nationale le 15 janvier 2012, énumérant les trois objectifs de cette intervention :

1. stopper l'offensive des groupes terroristes ;

2. préserver l'existence de l'État malien et lui permettre de retrouver son intégrité territoriale et sa totale souveraineté ;

3. préparer le déploiement de la force d'intervention africaine ${ }^{13}$.

Le rapport rappelle, que le même jour, en visite, à Dubaï, dans les Émirats arabes unis, François Hollande a employé des termes analogues pour qualifier l'intervention, en ajoutant de surcroît la nécessité de «sécuriser Bamako, où nous avons plusieurs milliers de ressortissants ${ }^{14}$, de «préparer, aider les forces maliennes à se structurer et s'organiser et [de faire en sorte] que la Misma puisse s'organiser pour aboutir à la reconquête totale du Mali ${ }^{15}$.

Paris n'a pas d'intérêt économique majeur au Mali, le pays ne disposant pas de ressources énergétiques. En revanche, les pays frontaliers sont stratégiques, notamment le Niger où Areva exploite l'uranium. Pour Les opposants à l'intervention française, la motivation réelle était la défense des intérêts économiques de la France dans la région, notamment les mines d'uranium du Niger, ou les ressources naturelles, souvent non encore exploitées, du Mali ou d'autres pays du Sahel. Jacques Attali insistait dans son blog sur la proximité géographique de la manne énergétique du Niger ${ }^{16}$.

Comme le rappelle Raphaël Granvaud, l'uranium qui alimente le nucléaire civil et militaire provient en grande partie du sous-sol africain. Cet auteur analyse les conditions dans lesquelles la France et Areva se procurent l'uranium au meilleur coût, au détriment des populations locales. L'intensification des concurrences marchandes sur le continent africain contraint Areva à s'appuyer sur l'État français. Il dénonce avec virulence «les réseaux les moins ragoûtants de la Françafrique pour sauvegarder son droit de pillage ${ }^{17}$. Le Niger, il est vrai, pèse pour $30 \%$ dans l'approvisionnement des centrales nucléaires françaises. Areva y possède deux mines, celles d'Arlit et d'Akokan ainsi qu'une troisième en construction à Imouraren. Cette dernière est censée produire 5000 tonnes de minerai et devenir la première mine d'uranium d'Afrique en 2020. Dans cette perspective, le Niger représenterait, à moyen

${ }^{13}$ Bernard Adam, Mali de l'intervention militaire française à la reconstruction de l'État, Bruxelles, GRIP, 2013/3, citation p. 10. Référence web : http://www.grip.org/sites/grip.org/files/RAPPORTS/2013/Rapport_20133.pdf. Page consultée le 12 septembre 2015.

14. Six mille Français sont présents au Mali.

15 . Vidéo du discours de F. Hollande sur : www.lemonde.fr, 15 janvier 2013. François Hollande, le discours de Bamako, 3 février 2013: https://www.youtube.com/watch?v=lXsz7IERYOs. Page consultée le 12 septembre 2015.

16. www.attali.com, 28/05/2012 : «les gisements d'uranium du Niger, essentiels à la France ne sont pas loin ». Page consultée le 12 septembre 2015.

17. Raphaël Granvaud, Areva en Afrique, la Face cachée du nucléaire français, Marseille, Éditions Agone, 2015. 
terme, la moitié des approvisionnements de la France. Après la prise d'otage meurtrière à In Amenas en Algérie, la France a décidé de sécuriser ses mines du Niger. La nouvelle mine d'Imouraren devrait atteindre des effectifs de plus de 300 employés expatriés français. Mahamadou Issoufou, le président nigérien, a accepté la présence de forces françaises sur son territoire, alors que son prédécesseur s'y opposait. Un article du Monde souligne que l'arrivée au pouvoir de François Hollande et de M. Issoufou a changé les relations entre les deux pays. Le président du Niger a soutenu l'intervention militaire française au Mali en envoyant directement des troupes à $\mathrm{Gao}^{18}$.

\section{«Préparée mais pas voulue », l'intervention française}

Dans leurs différents scénarios les militaires français avaient envisagé l'emploi des troupes africaines de la Cédéao, en appui à l'armée malienne, ainsi renforcée par les militaires européens. Les militaires français sur le terrain était conçue comme des soutiens essentiels mais non comme une force de libération directe, afin d'éviter de donner une image «coloniale» à l'intervention. Les plans esquissés avaient pour corollaire concret le positionnement de troupes françaises dans les pays voisins du Mali. Des témoignages font état du stationnement d'une dizaine d'hélicoptères de combat Gazelle et Tigre sur l'aéroport d'Ouagadougou au Burkina Faso fin décembre 2012. Le gouvernement n'a pas souhaité procéder à l'intervention à la date précise du 11 janvier 2013, comme le remarquaient des observateurs étrangers ; celle-ci a dû être précipitée face à l'offensive du 9 janvier vers le sud du Mali par les groupes jihadistes et à la demande d'aide du président malien. "C'est donc quelque peu contraints et forcés que les responsables français ont pris cette décision, note un conseiller du président français. François Hollande a tout fait pour ne pas y aller. C'est le dos au mur qu'il a accepté ${ }^{19}$. $»$ Aussi, très conscient de l'image négative de la tradition interventionniste en Afrique, l'Elysée se laisse-t-il entraîner à contrecœur dans une aventure à laquelle il a tenté de donner l'allure d'une opération en rupture avec les opérations antérieures de la Françafrique.

\footnotetext{
${ }^{18}$. http://www.lemonde.fr/afrique/article/2013/01/31/mines-d-uranium-la-france-n-a-pas-interet-a-ce-que-leconflit-malien-s-etende-au-niger_1825026_3212.html\#erQUWFIGbmO2me5U.99, Le Monde, 31 janvier 2013. Page consultée le 12 septembre 2015.

${ }_{19}$. « Guerre au Mali : comment l'Elysée a manœuvré », www.mediapart, 2 février 2013.
} 
Fig.3- Le territoire revendiqué par l'Azawad en avril 2012, crédit cartographique Agathe Plauchut

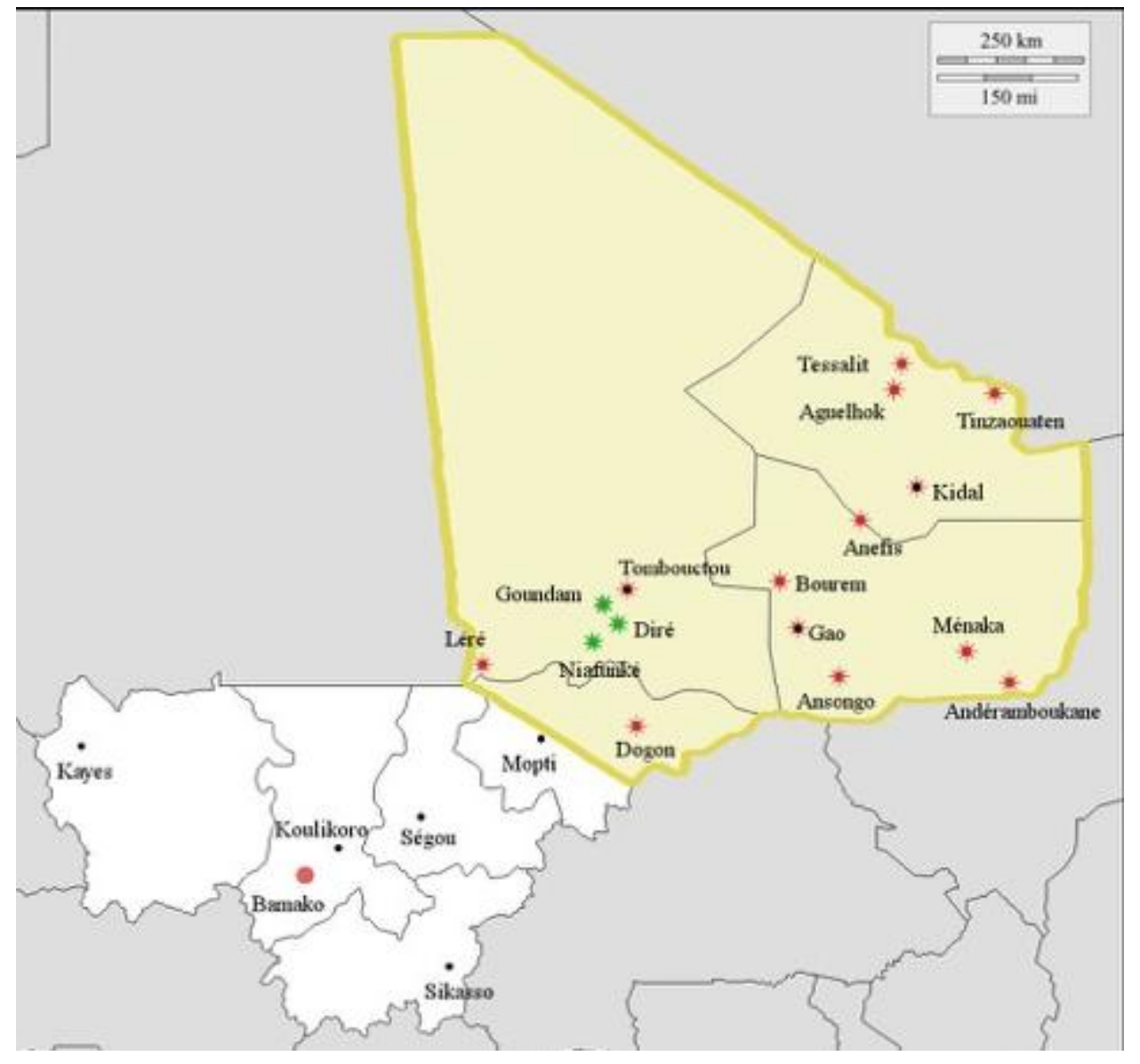

泋 Villes conquises, statut actuel incertain

夈 Villes actuellement occupées

Zone revendiquée

- Capitales provinciales 


\section{LE PASSÉ DU MALI POURRAIT-IL ÉCLAIRER LA CRISE ?}

\section{Des empires maliens au Soudan colonial}

Sur la crise malienne de nombreux spécialistes ont évoqué l'importance de se référer à la longue durée ${ }^{20}$. Comme chacun sait «la longue durée » en Histoire se rapporte à un construit braudélien. Pour cet historien, il était plus pertinent de se détacher de l'histoire événementielle «à oscillations brèves, rapides, nerveuses » pour insister sur la pertinence explicative de l'histoire quasi immobile qui s'intéresse aux phénomènes extrêmement longs (évolution des paysages, histoire de l'homme dans ses rapports avec le milieu).

Paradoxalement, les auteurs spécialistes de la question touarègue se référaient, en évoquant le temps long, au passé colonial, soit un passé très proche, ce qui la succession d'empires qui a longtemps dominé l'espace commercial transsaharien. Le Mali, colonie française de 1895 à 1960, subit quelques années après son indépendance la dictature de Moussa Traoré avant de se doter au début des années 1990 d'un régime démocratique. Dès lors, le pays, considéré comme un modèle de démocratie, ne répond pas à celui du Failed State qui ouvrirait la voie au terrorisme, selon certains historiens ou experts qui estiment que l'absence d'organisation étatique sert de brèche aux organisations terroristes : "La plupart des problèmes de sécurité en Afrique tiennent largement à la faillite de l'État $\operatorname{prostcolonial}^{21}$. » Le cas du Mali est un contre-exemple à opposer à cette théorie qui tend à établir des équivalences simples entre États faibles et terrorisme.

Bien que le Mali actuel soit une création coloniale, le Mali précolonial a été en fait l'épicentre d'empires florissants et un terreau fertile pour diverses formes étatiques. Berceau de trois grands empires médiévaux - l'empire du Ghana, l'empire du Mali et l'empire songhaï , ce territoire a expérimenté, sur la longue durée, une organisation étatique impériale. En effet le Ghana, le Mali, le Songhaï médiévaux ont élaboré, au fil du temps, des systèmes politiques sophistiqués en matière d'administration des communautés et de leurs territoires. Des peuples dits mélano-africains comme les Mandingues, les Bambaras, les Peuls du Mali, situés au sud, cohabitaient avec les nomades berbères du Sahara, les Touaregs, auxquels ils se sont souvent confrontés $^{22}$. Aussi le temps long pour l'histoire malienne devrait-il s'inscrire dans cette permanence impériale qui a façonné l'espace et les hommes bien avant la période coloniale.

Fondé par Sundiata Keïta au XIII ${ }^{\mathrm{e}}$ siècle, l'empire du Mali connut son apogée au XIV siècle (1320-1545). Sundiata Keïta est un personnage à la fois historique et légendaire dont l'épopée demeure aujourd'hui encore l'un des répertoires classiques des griots.

${ }^{20}$. Se référer aux travaux de Jan Jansen de l'université de Leiden, à ceux d'Hélène Claudot-Hawda et d'André Bourgeot.

${ }^{21}$. «Most of the security problems of Africa largely hang on the failure of the postcolonial state «: Sonia Le Gouriellec, «La menace stratégique des États Faibles : quand les faits relativisent la théorie », Note de recherche stratégique, $\mathrm{n}^{\circ} 18$, IRSEM, avril 2015 , citation p. 1 .

22 . Répartis et divisés en plusieurs confédérations et tribus, un million et demi de Touaregs vivent sur cinq pays. Ils seraient aux alentours de 1,5 million, dont 550000 au Mali, 800000 au Niger, 50000 en Algérie et au Maroc, le reste au Burkina Faso et en Libye. Les Kel Tamasheq, par exemple, ont traditionnellement ignoré les limites des États. La matrice originelle des Touaregs est berbère. Leur culture, fondée sur la langue tamasheq, est berbère. «L'appellation «Touareg » est d'origine arabe. L'ethnonyme des populations ainsi désignées est, selon les variantes locales, Kel Temacheq, Kel Tamajeq, Kel Tamahaq, c'est-à-dire «ceux qui parlent la langue tamacheq ». Celle-ci appartient à l'ensemble linguistique berbère ». André Bourgeot, op, cit, p.131. André Bourgeot, «Identité touareg : de l'aristocratie à la révolution », Études rurales, n¹20, 1990. Identité, p. 131. L'islam se propage à travers le Sahara au $\mathrm{XI}^{\mathrm{e}}$ siècle à partir du Maroc. 
La dynastie des Keïta régna, sans interruption, jusqu'en 1389. Mansa Moussa (1312-1332), le $10^{\mathrm{e}}$ empereur du Mali, fut considéré comme l'un des rois les plus riches de son époque. Il établit des relations diplomatiques régulières avec le Portugal, le Maroc, la Tunisie et l'Égypte. Le territoire du roi des rois allait du Sahara et de la forêt équatoriale jusqu'à l'océan Atlantique et la boucle du Niger, recouvrant les actuels Mali, Burkina Faso, Sénégal, Gambie, Guinée, Guinée-Bissau, Mauritanie et une grande partie de la Côte d'Ivoire. L'empire se composait d'une constellation de confédérations d'États tributaires. Les provinces étaient administrées par des gouverneurs appelés farins ou farba. L'empereur (mansa) était assisté par un conseil des anciens (chefs militaires, civils et marabouts). L'Empire était prospère grâce au commerce transsaharien et aux richesses minières (or et cuivre). Les centres urbains, Oualata, Tombouctou, Djenne et Niani, la capitale, étaient florissants.

La centralité du pouvoir a donc une origine ancienne au Mali : à l'époque médiévale, la clé de voûte du système politique était le roi des rois ou mansa, personnage tout puissant au pouvoir seulement tempéré par les coutumes ancestrales et les prescriptions de l'islam. Des traditions orales transmises par les griots mandingues et les historiens arabes nous ont largement documentés sur cet empire, notamment le damascène Al Omari (1304-1348), Ibn Battouta (1304-1377), originaire de Tanger. Le royaume sonrhaï de Gao parvint à détruire l'empire du Mali au milieu du XV $\mathrm{X}^{\mathrm{e}}$ siècle.

A la fin du XVe siècle, l'empire du Mali céda la place à l'empire sonrhaï avec Gao et Tombouctou comme cités principales. , puis une armée envoyée par le sultan du Maroc mit fin à l'empire sonrhaï en 1591. Un nouvel empire Bambara a vu le jour au milieu du XVII siècle autour de Segou. Ce royaume animiste a été érigé par Kaladjan Coulibaly qui y régna en maître pendant trente ans (1652-1682). En 1861, El Hadj Oumar Tall s'empare de Ségou et remplace le royaume Bambara par le royaume Toukouleur qui, au $\mathrm{XIX}^{\mathrm{e}}$ siècle, est une théocratie musulmane. Son fils et successeur, Amadou Tall ne parvient pas à maintenir l'unité de son royaume. Lorsque les Français conquièrent Ségou en 1890, Amadou doit fuir à Sokoto, au nord du Nigeria. En faisant ainsi voler en éclats une structuration politique qui s'enracinait profondément dans le passé, la puissance coloniale avait-elle réellement fait disparaître les aspirations impériales et la volonté de puissance des anciens royaumes Bambara et Toukouleur ? On est en droit d'en douter.

La conquête française met donc fin au royaume Toukouleur. Elle fut l'œuvre de militaires successeurs de Faidherbe, qui, à partir du Sénégal, s'avancèrent progressivement vers l'est. La colonie française porte le nom de Soudan entre 1891 et 1904 avant d'être intégrée, entre 1920 et 1946, à l'Afrique occidentale française (AOF), érigée sur le territoire de l'actuel Mali. Ces territoires ont constitué la colonie du Haut-Sénégal-Niger en 1904, avec d'abord Kayes sur le fleuve Sénégal pour capitale, puis Bamako occupée en 1883 et chef-lieu de cercle en 1895.

\section{Les Touaregs}

L'organisation politique des Touaregs n'a jamais été structurée autour des notions de royaume, de roi et d'empire. Leur vision politique s'organise autour de la notion de tribu. Leur organisation politique précoloniale a été décrite par Hélène Claudot-Hawad ${ }^{23}$. Les «tribus » (tawshit au singulier), chapeautés par un aîné (amghar), sont rassemblées en confédérations ou ettebel $^{24}$. Le chef de la confédération était appelé ettebel (chef à tambour de

\footnotetext{
23. Hélène Claudot-Hawad, «Honneur et politique : les choix stratégiques des Touaregs pendant la colonisation française », Revue du monde musulman et de la Méditerranée, $\mathrm{n}^{\circ}$ 57, 1990. pp. 11-48.

${ }^{24}$. Littéralement : tambour, symbole du pouvoir politique chez les Touaregs.
} 
commandement) ou amghar. «Le schéma égalitaire de la tawshit recouvrait en pratique une situation inégalitaire entre des familles dominantes qui accordaient leur protection à des groupes plus faibles en contrepartie d'un tribut ou d'une allégeance politique ${ }^{25}$. $\gg$. Le jeu de pouvoir complexe entre les familles se trouvait également régulé par les alliances matrimoniales.

Les Touaregs à l'époque coloniale se heurtent violemment aux colonisateurs sur une base inégalitaire car les guerriers touaregs ne possèdent pratiquement pas d'armes à feu.

[Dans les premières années du $\mathrm{XX}^{\mathrm{e}}$ siècle,] toutes les confédérations ont essuyé de lourdes défaites et ont dû faire leur soumission, à l'exception des Kel Ajjer. [...] Pour la première fois dans l'histoire du pays touareg, nous dit Hélène Claudot-Hawad, l'ensemble de la société est atteint et son équilibre remis en question. L'aspect jugé le plus terrible de ces défaites militaires réside en effet dans leur extension à tous les fronts que constituent les confédérations touarègues, du nord au sud et de l'est à l'ouest. Si l'affaiblissement d'un élément de la charpente est considéré comme naturel, l'effondrement, en moins de dix ans, de tous les piquets crée une catastrophe sans précédent qui conduit à la condamnation et à l'extinction de la société tout entière ${ }^{26}$.

Dans la littérature coloniale les mots qui accompagnent le plus communément celui de «Touaregs» sont ceux d' «anarchie » et de «pillage». Le monde colonial jugeait les populations touarègues «ingouvernables». Elles sont assimilées aux «rezzous » et leur société réduite à une organisation de pillards ${ }^{27}$. Ces stéréotypes coloniaux semblent fixés dans l'imaginaire : d'aucuns ont pu interpréter la crise malienne de 2012 comme « le retour des rezzous $»^{28}$.

Force est de constater que l'organisation sociale des Touaregs, flexible et décentralisée, se situe aux antipodes du modèle jacobin. Or, le colonisateur français met en place au Mali un système très centralisé administré par les chefs de cantons. Un arrêté du gouverneur général crée le $1^{\text {er }}$ janvier 1911 des communes mixtes. Les premières sont constituées à Bamako et Kayes le $1^{\text {er }}$ janvier 1919, puis à Mopti au $1^{\text {er }}$ janvier 1920. Dans un second temps, on créa en 1953 et 1954 les communes mixtes de Ségou et Sikasso. Ces unités administratives sont gérées par un maire nommé par arrêté du lieutenant-gouverneur, assisté par une commission municipale du $1^{\text {er }}$ degré composée de huit membres titulaires (quatre notables citoyens français, quatre notables sujets français) et quatre membres suppléants (deux citoyens français, deux sujets français). La loi française ${ }^{\circ} 55-1489$ du 18 novembre 1955 prévoit la création de communes de plein exercice - par décret pris sur les rapports du ministre de la France d'Outre-Mer - après avis de l'assemblée territoriale intéressée. Un conseil municipal est élu par un collège unique qui désigne un maire en son sein. Par cette même loi, Bamako, Kayes, Ségou et Mopti deviennent en 1956 des communes de plein exercice. Sept communes de moyen exercice sont créées en 1958: celles de Kita, Kati, Koulikoro, Koutiala, San, Tombouctou et Gao. Le maire est un fonctionnaire nommé par le chef de territoire ; il est assisté par un conseil municipal élu par un collège

25. Clare Oxby, «Les allégeances politiques d'une "tribu” touarègue entre deux ex-confédérations (Kel Ferwan, Dakoro, Niger)», in Hélène Claudot-Hawad (dir.), Touaregs et autres sahariens entre plusieurs mondes, Aix-en-Provence, EdiSud, 2014, pp.170-182. Référence web : http://books.openedition.org/iremam/2852?lang=fr. Page consultée le 12 septembre 2015.

26. Hélène Claudot-Hawad, «Honneur et politique ... », op. cit., citation p. 23.

${ }^{27}$. Le terme « rezzou» se rapporte à une incursion rapide en territoire étranger, dans le but de prendre le butin. Le mot provient de l'arabe gazwa (غزو) : raid ; invasion ; conquête.

${ }^{28}$. Le document publié sur internet : Christian Roche, Regard sur l'Histoire du Mali (non publié), avait pour titre «le retour des rezzou ». Référence web : http://aphglyon.free.fr/regards-sur-1-histoire-du-mali.pdf. Page consultée le 12 septembre 2015. 
unique. Cette organisation permet de saisir toute l'ampleur d'un squelette administratif français hybride qui a recyclé un découpage impérial ancien.

Comme ailleurs dans d'autres territoires coloniaux, une nouvelle classe d'administrateurs a émergé de cette organisation administrative de type cantonal centralisateur. Les maires imposés par les Français ne sont pas validés par les populations locales ; cette chefferie cantonale à la française incarne un nouveau système coercitif, comme le remarque l'anthropologue Bréhima Kassibo. L'administration française, nous di-il, a utilisé l'ancien découpage territorial des empires maliens tout en y installant de nouveaux administrateurs :

[Elle] s'est greffée sur l'ossature de ces anciennes organisations territoriales : province ou région (jamana), canton (marabolo oukafo), village (dugu), du moins dans ce qu'on peut qualifier d'anciennes aires culturelles marka, mandé, songhaï et peule. La politique des races, appliquée par l'administration coloniale française au lendemain de la «pacification» du pays, s'est orientée vers la promotion des leaders politiques traditionnels auxquels elle octroyait le droit d'administrer leurs circonscriptions en tant qu'auxiliaires désignés, au détriment des opposants politiques locaux. Il en a résulté un renforcement des prérogatives de ces nouveaux chefs, que la coutume ne leur reconnaissait point et qu'elle s'était toujours évertuée à contrôler à l'aide d'organes de régulation appropriés (conseils de village, d'arbitrage, de conciliation). Ainsi, les populations furent-elles intégrées dans un vaste système coercitif (travaux forcés, conscription, brimades) dont l'exécution reposait principalement sur la chefferie cantonale. Juché au sommet de cette pyramide, le commandant blanc de cercle ou de subdivision demeurait l'épicentre des prises de décision capitales. Il ajoutait à ces prérogatives administratives, celles judiciaires et de police, signes évidents de son omnipotence arbitraire. C'est cette organisation coercitive de l'administration coloniale qui s'est incrustée dans la mémoire collective et a estompé dans les esprits le souvenir des systèmes d'administration précoloniaux dont certains faisaient preuve de plus de souplesse dans leur application ${ }^{29}$.

\section{Rebellions touarègues à répétition}

Cet héritage colonial a influé sur le tracé des frontières, et la centralisation de type jacobine à partir de Bamako n'a fait que renforcer les rébellions touarègues. On compte au moins six grandes révoltes touarègues depuis 1916. La crise malienne de 2012 s'inscrit dans ce continuum de résistance et d'irrédentisme auquel l'indépendance du pays n'a apporté aucune solution. À la rébellion de 1916 au Niger succède celle de 1962-1964, très durement réprimée par l'armée malienne, puis celle de 1990-1995 au Mali et au Niger. Au Mali, ce conflit débouche sur les accords de Tamanrasset de 1991 et le pacte national de 1992. Les cessez-le-feu ne se soldent cependant pas par la fin d'hostilités. Les braises du conflit sont rallumées en 1994-1995 pour paraître s'éteindre en 1996 à la faveur d'accords de paix signés à Tombouctou. Mais le feu reprend à nouveau, le 23 mai 2006 ; le soulèvement se conclut par des accords signés à Alger le 4 juillet 2006, qui, à nouveau, n'apportent qu'une courte trêve. La rébellion repartit en 2007-2009.

Depuis 1958, date de la création du mouvement populaire de l'Azawad, les Touaregs revendiquent, en vain, la constitution d'un État touareg, problème que la crise de 2012 n'est pas parvenue à résoudre. Les Touaregs renoncent finalement à leur ambition territoriale le 14 février 2013. Aucun gouvernement ne soutient leur cause et ne reconnaît la légitimité de leurs aspirations territoriales. Le terme azawad qui signifie «zone de pâturage» vient du mot tamasheq Azawagh. Il exprime le cœur de la revendication d'un peuple privé de ses terres

${ }^{29}$. Bréhima Kassibo, « La décentralisation au Mali : état des lieux », Bulletin de l’APAD, n $14,1997$. Référence web : apad.revues.org/579. Page consultée le 12 septembre 2015. 
et de son ambition : créer une nation touarègue au Nord Mali. Cette quête d'État, engagée depuis la période coloniale, a été étouffée par les équilibres politiques postcoloniaux.

Or, Les référents lignagers sont en relation étroite avec le territoire : «Les règles résidentielles, les modes d'alliance matrimoniale, les rapports aux troupeaux, les connaissances écologiques, etc., n'ont plus de pertinence sociale et ne participent plus à la formation de la personnalité ethnique ${ }^{30} \ldots$ » Les identités sociales sont liées à l'appartenance à un territoire. Dans le film ethnographique d'Hélène Claudot-Hawad, Furigraphier le vide $e^{31}$, le poète Hawad exprime de façon métaphorique l'identification de l'homme à chaque pierre de l'espace en question, soulignant la symbiose entre les espaces réel et imaginaire, permettant de saisir l'osmose entre revendication identitaire, politique et territoriale. Le renoncement aux terres implique une forme de renoncement à soi. De crise en crise, le monde touareg s'est paupérisé, marginalisé voire « criminalisé ». Le film nous montre une poésie du désespoir qui tente par la langue, dernier patrimoine, de créer un territoire de mots ${ }^{32}$. Du point de vue de la revendication touarègue, l'opération «Serval » de 2012 pourrait apparaître, ainsi replacée dans la longue durée, comme une crise de plus dans une série qui remonte à 1916. Il s'agit donc bien d'une crise chronique. C'est dans cette optique que des observateurs ont pu interpréter la crise malienne de 2012 comme l'éternel "retour des rezzous ». L'alliance des Touaregs avec Aqmi ou le Mujao, en 2012, serait à interpréter, en quelque sorte, comme un accident de parcours. Cette alliance relève de calculs stratégiques temporaires trouvant leur raison d'être dans une volonté tenace d'indépendance touarègue. Car tout oppose les organisations salafisto-terroristes, fondamentalement rigides, à la flexibilité et à la mobilité de la cosmogonie touarègue.

L'irrédentisme au long cours des Touaregs renvoie à la crise du Darfour (2003-2009) et à la revendication d'autonomie de cette province soudanaise sauvagement réprimée par Khartoum. La crise du Darfour comme la crise malienne sont «endémiques » en ce sens que leurs ferments reposent sur des revendications territoriales, nationales, identitaires profondes qui s'inscrivent dans la longue durée. Le mot «crise » nous vient du grec ancien (krisis) et, étymologiquement parlant, le concept induit le sens de « décision » et de « jugement » entre deux choix possibles. Il incarne l'insolite, l'instabilité et une situation de rupture. Aussi, lorsque l'état de krisis s'inscrit dans la durée, convient-il de parler de "crise chronique ». L'espace-temps de la crise est une donnée essentielle pour discerner chez elle un paradigme de crise permanente. La crise du Nord Mali s'inscrit dans le long terme et la notion de guerre civile serait plus appropriée pour caractériser les évènements. La médiatisation du conflit en Occident n'intervient qu'en 2012, mais la crise est là, menaçante, depuis les années 1916, telle une savane asséchée prête à s'embraser au moindre "coup de soleil». Edgar Morin souligne avec pertinence que le terme de crise a été tant galvaudé qu'il est aujourd'hui vidé de son sens premier, un contenu qui, nous l'avons dit, rappelait la notion de décision, de choix. Or, l'histoire du $\mathrm{XX}^{\mathrm{e}}$ siècle a fait évoluer la signification du mot vers une notion d'indécision, d'incertitude ${ }^{33}$. En conséquence, les crises évoquées dans notre ouvrage Humanitarian crises sont des exemples de crises durables, permanentes qui ont transformé le provisoire en une chronicité inédite, telle une maladie dont les symptômes apparaissent lentement, durent longtemps et s'installent même parfois définitivement ${ }^{34}$.

\footnotetext{
${ }^{30}$. André Bourgeot, op. cit., pp. 129-162, citation p. 140.

${ }^{31}$. https://www.canalu.

tv/video/universite_toulouse_ii_le_mirail/furigraphier_le_vide_art_et_poesie_touareg_pour_le_iiie_millenaire_h elene_claudot_hawad.10202 Page consultée le 12 septembre 2015.

32. André Bourgeot, op, cit, p. 131.

33. Edgar Morin, op. cit., pp. 149-163.

34. Fabienne le Houérou, Humanitarian Crises and International Relations 1959-2013, Sharjah (EAU), Bentham Science Publishers, 2014.
} 
La crise du Darfour a été décrite par certains journalistes et quelques intellectuels à la marge du monde universitaire comme un conflit «ethnique », en réduisant la guerre à une haine «tribale», et en opérant une distinction catégorique entre nomades «arabes » et sédentaires « africains », réinventant des projections datant souvent de l'époque coloniale. Cet essentialisme ethnique, réapparu en 2012 avec la crise malienne, oppose les Arabo-Berbères aux Négro-Africains. C'est ainsi que le passé peut ressurgir dans les lunettes d'observation des médias occidentaux. Or, la crise humanitaire du Mali, conséquence d'un conflit meurtrier, réunit toutes les composantes d'une question qu'il faut aborder avec une logique de complexité. Les facteurs explicatifs s'enchevêtrent savamment. Conjuguer la dimension écologique et économique au chaos social s'impose pour comprendre les imbrications de cette crise et ses strates empilées. Aussi, au regard d'une observation des grandes crises des $\mathrm{XX}^{\mathrm{e}}$ et $\mathrm{XXI}^{\mathrm{e}}$ siècles, semble-t-il important pour une théorie moderne des relations internationales que nos réflexions épistémologiques s'inspirent de celles d'Edgard Morin sur la pensée complexe $^{35}$. Ce dernier nous invite en effet à abandonner le paradigme explicatif unique, « le paradigme perdu», par exemple le paradigme de l'État faible «failed», «failli», et préconise, pour comprendre les conflits ou les crises qui ne sont humanitaires que d'apparence, de mettre en œuvre un savoir et une pensée qui respectent le caractère pluridimensionnel des crises. Il nous invite à associer des notions qui s'excluent, prônant une « crisiologie » de l'interdépendance qui se ramène à une approche « encyclopédique » au sens classique de la connaissance.

La crise malienne s'apparente ainsi à de nombreuses crises africaines qui ne sont pas seulement le produit d'États postcoloniaux faibles mais qui conjuguent une série de facteurs. La tradition française de la Françafrique y tient encore un rôle important. D'aucuns auront décrypté, dans la crise malienne, l'éternel retour des habitus d'une Françafrique que l'on avait cru définitivement révolue. Tout se passe comme si les bonnes intentions ne parvenaient pas à se détacher d'une mécanique internationale où la France demeurait le «gendarme » malgré elle. Le rôle d'arbitre est certes un legs colonial marquant au sol la continuité d'une raison d'État en contradiction avec les exigences morales et éthiques d'un pouvoir qui se voudrait irréprochable mais néanmoins affecté par la réalité entêtante des faits.

Fabienne LE HOUÉROU

Migrinter UMR 7301, CNRS/Université de Poitiers

\footnotetext{
${ }^{35}$. Edgard Morin, Introduction à la pensée complexe, Paris, Le Seuil, 2005.
} 